EPiC Series in Engineering
Volume 3, 2018, Pages 676-683
HIC 2018. 13th International
Conference on Hydroinformatics

\title{
SIM4NEXUS - Coupling a System Dynamic Model with Serious Gaming for policy analysis
}

\author{
Barry Evans ${ }^{1 *}$, Lydia Vamvakeridou-Lyroudia ${ }^{1}$, Janez Susnik ${ }^{2}$, Antonio \\ Trabucco $^{3,4}$, Simone Mereu ${ }^{3,4}$, Xavier Domingo ${ }^{5}$, Chengzi Chew ${ }^{6}$ \\ and Dragan Savic ${ }^{1}$ \\ ${ }^{1}$ Centre for Water Systems, University of Exeter, Exeter, EX4 4QF, United Kingdom \\ ${ }^{2}$ IHE Delft Institute for Water Education, PO Box 3015, 2601 DA Delft, Netherlands \\ ${ }^{3}$ University of Sassari, Department of Science for Nature and Environmental Resources, Sassari \\ Italy \\ ${ }^{4}$ Euro-Mediterranean Center on Climate Changes, IAFES Division, Sassari, Italy \\ ${ }^{5}$ EURECAT, Lleida Agrifood Science and Technology Park, ICT Building, Lleida 25003, Spain \\ ${ }^{6}$ DHI, Agern Alle 5, Hørsholm 2970, Denmark \\ b.evans@exeter.ac.uk
}

\begin{abstract}
Water, land, food, energy, and climate are all interconnected, comprising a coherent system (the 'Nexus'), dominated by complexity and feedback. The interactions between these different nexus components and their responses to climate change conditions are complicated as each feedback into the other. Consequently future policies should take into account the whole Nexus, when it comes to ascertain their long term impact on the system. This paper presents the conception of a System Dynamic Model (SDM) representing the Nexus, populating it with data from various sources (including output from specific thematic models covering different Nexus components), under different climate change and socioeconomic pathway scenarios. The SDM is then converted into $\mathrm{R}$ scripting to be included in the Knowledge Elicitation Engine (KEE) communicating with a Serious Game (SG). Models and games are built specifically for ten Case Studies, at regional, national, continental and global level. The SG is being developed for decision making for local stakeholders to study and get acquainted to long term impacts of different policies on the Nexus. In this paper the regional Case Study of Sardinia is presented as proof of concept.
\end{abstract}

${ }^{*}$ Corresponding author 


\section{Introduction}

Land, food, energy, water and climate are interconnected, comprising a coherent system (the 'Nexus'), dominated by complexity and feedbacks (Mohtar \& Daher, 2010). Putting pressure on one part of the Nexus can generate pressures on another. Management of the Nexus, i.e. integrally addressing all these sectors rather than 'fixing' one and exacerbating the others, is critical to securing the efficient use of our scarce resources. Changes to one aspect within the Nexus can both directly and indirectly affect other components and potentially feedback onto itself within a positive or negative feedback loop. A Nexus based approach will not only show the negative consequences but would also highlight potential synergies which are harder to grasp. This would allow policy makers to work in a positive way looking for synergies as opposed to just identifying conflicts. The same happens with most existing simulation models, which support/simulate some of the Nexus components, but not all of them. The ongoing H2020 SIM4NEXUS project (www.sim4nexus.eu) aims to help rectify this issue via the use of a System Dynamic Modelling (SDM) approach, developed for systems at regional, national level, which then feeds into a Serious Game (SG) simulation engine. The SG can be subsequently used by decision makers and local stakeholders to try out and test long-term implications of different policies. Table 1 shows the regions being investigated during the course of the SIM4NEXUS project and their corresponding scales.

\begin{tabular}{lr}
\hline Location & Scale of Case Study /25 \\
\hline Andalucía & Regional \\
Azerbaijan & National \\
Eastern Germany-Czech Republic-Slovakia & Transboundary \\
France-Germany & Transboundary \\
Greece & National \\
Latvia & National \\
Netherlands & National \\
Sardinia & Regional \\
South west of United Kingdom & Regional \\
Sweden & National \\
& \\
Europe & Continental \\
Global & Global \\
\hline
\end{tabular}

Table 1: Scale and location of Case Studies being examined in SIM4NEXUS project

The Case Study for Sardinia is presented here, where, based on results from previous studies (Mereu et al., 2016), SDM is being used to calculate the operational resilience of reservoirs subject to climate change, taking into account different competing water uses, i.e. agriculture, hydropower, tourism and urban demands. This paper looks to expand upon this research to incorporate additional factors into the Nexus model and allowing for the effects of policy changes by stakeholders to be examined against future climate change scenario predictions through the use of a SG.

\section{Methodology}

Within this research a framework was developed that describes the work flow for the creation of the SDM to represent the Food-Water-Energy nexus that can be fed into a SG to facilitate policy making decisions for the future under possible climate change scenarios. 


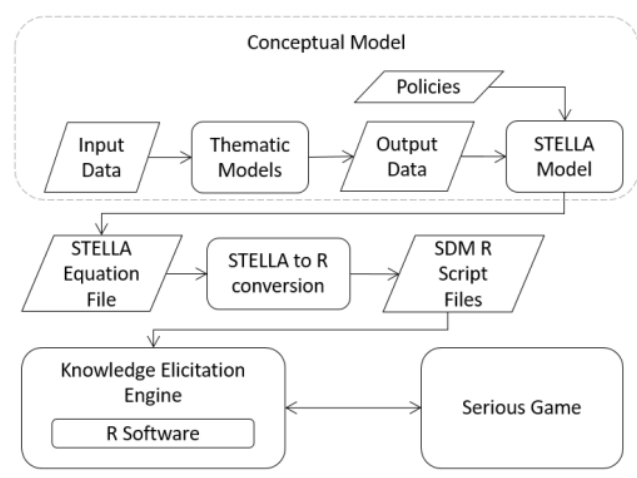

Figure 1 - Methodological workflow diagram

Figure 1 depicts the steps taken within the workflow from the Conceptual Model all the way through to the SG. Here the Conceptual Model is the main driver in the creation of the SDM. Policies can be implemented and modelled according to the following sequence: Conceptual Model (with Thematic

Models as input sources) $\rightarrow$ SDM (with Policy consideration) $\rightarrow$ Converted R Script $\rightarrow$ communication with Knowledge Elicitation Engine $\leftrightarrow$ Serious Game.

\subsection{Conceptual Model}

The first stage in this process is to design a conceptual model representation of the nexus with respect to the study area. The conceptual model is designed to be visual representation of the nexus framework whereby links (fluxes and connectors) between various components are defined in a clear manner and the drivers of those components from the thematic models can be considered. Figure 2 shows the initial derived nexus framework for the Sardinia case study based around availability of resources (water and energy) and demand from different key sectors (agriculture, domestic, industrial touristic) to grant specific "sector security" and interact by interlinks of "Thematic processes" where "climate dependencies" are highlighted to show impact of climate change on the Nexus.

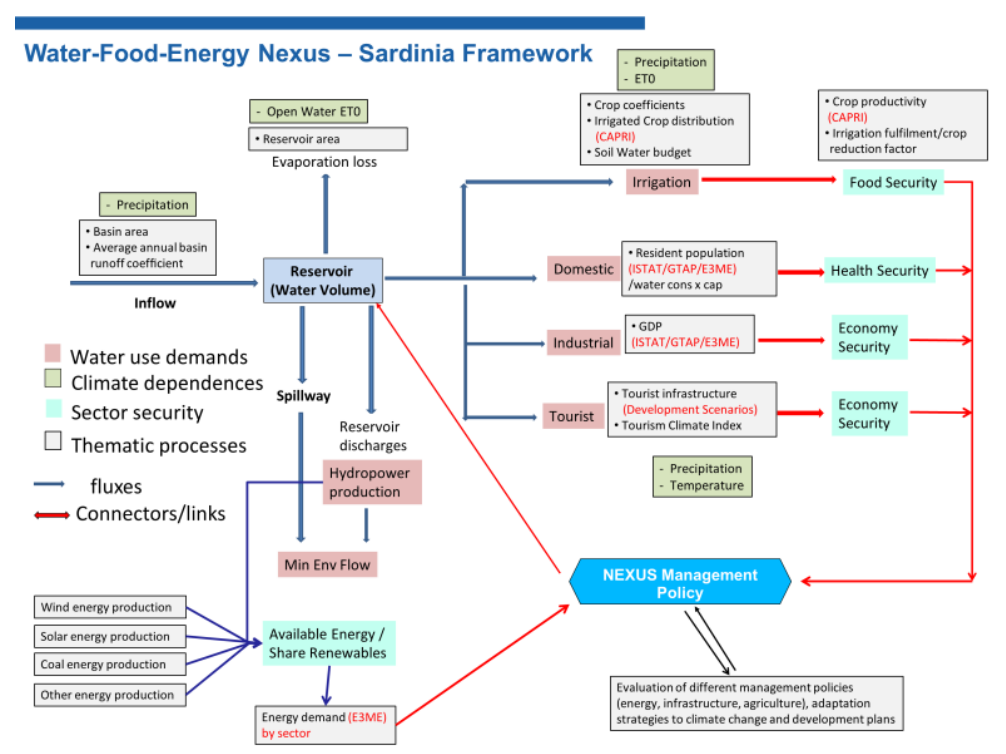

Figure 2: Conceptual model of the Water-Food-Energy NEXUS for Sardinia 


\subsection{Thematic models}

The thematic models used in SIM4NEXUS provide data/predictions for different parts/components of the Nexus that are then incorporated and utilised within the SDM. These thematic models are run separately for the case study area to produce time-series data based on entities such as irrigated area, changes in GDP and changes in energy uses per sector for future forecasts, e.g. 2010 (Baseline), 2020, 2030, 2040, and 2050.

Based on the above conceptualization, it was possible to identify four relevant 'thematic models' that feed information into the Sardinian SDM, from which derive needed data on present and projected future trends:

- CAPRI (a global agricultural and production model; ("CAPRI (Common Agricultural Policy Regionalised Impact Modelling System),” 2017) for irrigated area by crop

- $\quad$ E3ME (a global economic and energy model; (“E3ME: Our Global Macro-econometric Model," 2017) for energy production and demand by sector

- GTAP project database (www.gtap.agecon.purdue.edu/) for several socio-economic factors (e.g. population growth, GDP by sector) ("GTAP (Global Trade Analysis Project)," 2017)

- $\quad$ ISI- MIP for downscaled climate data (Hempel, Frieler, Warszawski, Schewe, \& Piontek, 2013)

All other data are from local Sardinian sources, as well as locally relevant data (e.g. for reservoir operating rules and environmental flow regulations).

\subsection{System Dynamic Model}

Based upon the Conceptual Model an SDM is built using Stella Architect ${ }^{\circledR}$ (www.iseesystems.com). During the development of the SDM the data obtained via the thematic models and climate projections are included, and the initial state of variables, such as reservoir levels and population, are defined. The SDM itself and the interaction between its components behave as a large time-dependent and condition-dependent partial differential equations. It is the conditional components that provide the means of defining policies that govern the subsequent behaviour of the model over time. For this case study there are seven different policies for the potential users: Minimum Environmental Flows, Efficiency of the water distribution systems, Priorities under water scarcity, Methane, Energy independence of the water authority (ENAS), Tourism, and Renewable energies. As an example, for the Minimum Environmental Flows the goal is to guarantee the sustainable functioning of the ecosystems downstream of the reservoirs by allowing minimal environmental flows, thereby conserving biodiversity and ecosystem balance. For this, the player can select 3 alternative policies types:

1. Foster biodiversity conservation and wetland productivity (fisheries)

2. Prioritize water demands for the Domestic (Tourism) and Agriculture sector

3. Increase resilience to climate change for the ecosystems downstream the reservoir

Here the $\%$ of reservoir volume is used as a threshold for controlling/reducing minimum environmental flows. 


\subsection{Policy Implementation within the SDM}

The basic setup of the SDM provides the framework for the flows and interactions of the thematic models where the response to future climate scenarios as Business As Usual (BAU), i.e., no action, can be analysed. However the creation of an SDM needs to consider how policies can be incorporated and applied within the model, and this step therefore needs to be considered during the conceptual stage. As highlighted in the previous section, the SDM functions as both time and condition dependent equations. Therefore via the incorporation of extra conditional statements and parameters, the effects of policy changes can be encapsulated. The initial (default) policy in the SDM that determines the volume of water released as environmental flow is as follows:

- If water in the reservoir is above $20 \%$ of the reservoir capacity then $10 \%$ of the monthly basin run-off will be released.

- If water in the reservoir is below $20 \%$ of the reservoir capacity then $5 \%$ of the monthly basin run-off will be released.

- If water in the reservoir is below $10 \%$ of the reservoir capacity then $0 \%$ of the monthly basin run-off will be released.

Thus the flow of water to the environment may be limited to zero, during times of significant drought (low reservoir volume). In the case of fostering biodiversity conservation and wetland productivity (fisheries) however the policy requires water to be supplied (to some degree) at all times with the rules now stipulating:

- If water in the reservoir is above $20 \%$ of the reservoir capacity then $20 \%$ of the monthly basin run-off will be released.

- If water in the reservoir is below $20 \%$ of the reservoir capacity then $10 \%$ of the monthly basin run-off will be released.

This conservation policy can be referenced within the STELLA model via the use of IF ELSE statements in conjunction with a "BiodiversityPolicy" switch; thus it is coded (Figure 3) within the STELLA environment as follows:

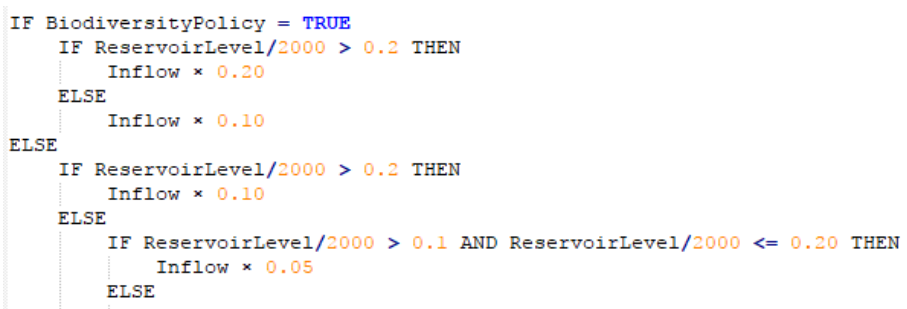

Figure 3: Representing policy control within a section of the SDM equation file

\subsection{R Conversion Script}

To bridge the gap between the SDM and the Knowledge Elicitation Engine, the SDM is converted to an R script via the use of a Python script, behind the SG platform the formula view. Due to the complex nature and variability in the way SDM models/equations can be written in terms of their parameters and functions, the Python script carries out multiple parses to firstly mine the parameters and then ensure that they ordered in such a way that the R script can interpret the created code correctly. The generated R Script is divided up into five parts: (1) Main R Script, (2) Function Components, (3) 
Time Series data files, (4) Time series Coefficient Values, and (5) Variable list and values. The R Script is integrated in the KEE and exposed using a Service Oriented Architecture, allowing the SG user interface for interaction. The first three components constitute the SDM whereas the fourth and fifth components are editable via the SG interface, to allow for policy changes. For instance threshold values for water scarcity levels or percentage reduction components of environmental flow as a result of water scarcity can be modified in this way.

\subsection{Serious game}

The SG (Figure 4) represents the front end that provides an interactive means for stakeholders and policy makers to see the implications their policies could have on the Nexus, whilst simultaneously hiding the complexities that go on behind the scenes, within the modelling deriving the information. The aim of the SG is to allow the discovery of various combinations of policies across the Nexus to achieve the objectives of each particular case study while providing also an evaluation about how nexuscompliant the combination of policies are.

In detail, when payers start a game session, they select a scenario (case study), and the desired role they want to play in the game. Depending on the role the player selects, different options (policies) will be available. The main underlying objective of the SG is to learn while playing. This means allowing the player to understand which the consequences are of making a decision (i. e. applying a policy) along time, so as to see the evolution of the different indicators, and how the various elements on the Nexus are affected. To that end, the SG is designed as a game by turns. On each turn, each player is able to see the current status of the scenario and check how close or far they are from achieving the scenario goals. Hence, considering the current status, the player decides to apply one or more policies (or stop applying one or more of them), and ends the turn. In turn, the SG asks the KEE which is the evolution of the scenario status, which is calculated by running the SDM model corresponding to the selected scenario. However, as the SDM model has to take into consideration the currently applied policies, the KEE translates the applied policies to weights in SDM input parameters, so as to run the selected combination. Result are then obtained and presented to the player, who starts the cycle again until achieving the desired scenario goals or ending the game.

It is very important to point out that by this process the player learns while playing, as the consequences of actions and selections can be easily understood. Moreover, a graphic user-friendly interface makes the experience more satisfactory, while navigating for information is much easier.

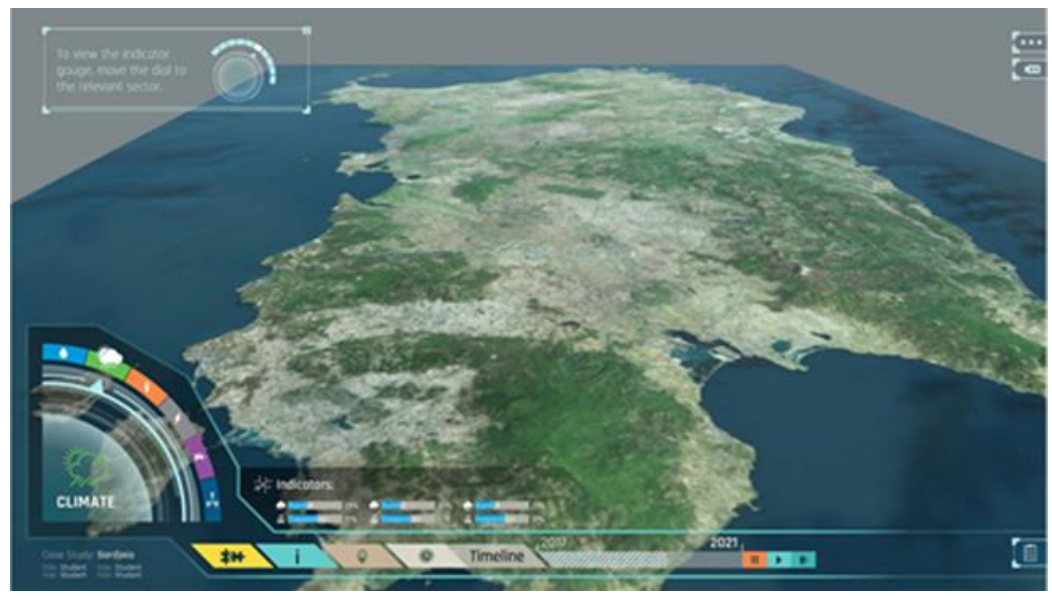

Figure 4: Screenshot of Serious Game interface 
One of the novel features within the SG is the JSON tree based approach (Figure 5) used to visualise changes components over time within the nexus as a result of policy implementation. Here the model of the nexus is depicted as an interconnected branch and node based structure, where the values that dictate changes to a given node are shown as the branches that come off that node. In the example shown in Figure 5, the influences of components that are driving water levels within the reservoir (dReservoir) in the model are shown by the chain underneath, where each preceding node that is derived from other variables can be expanded upon, showing their underlying drivers/dependencies.

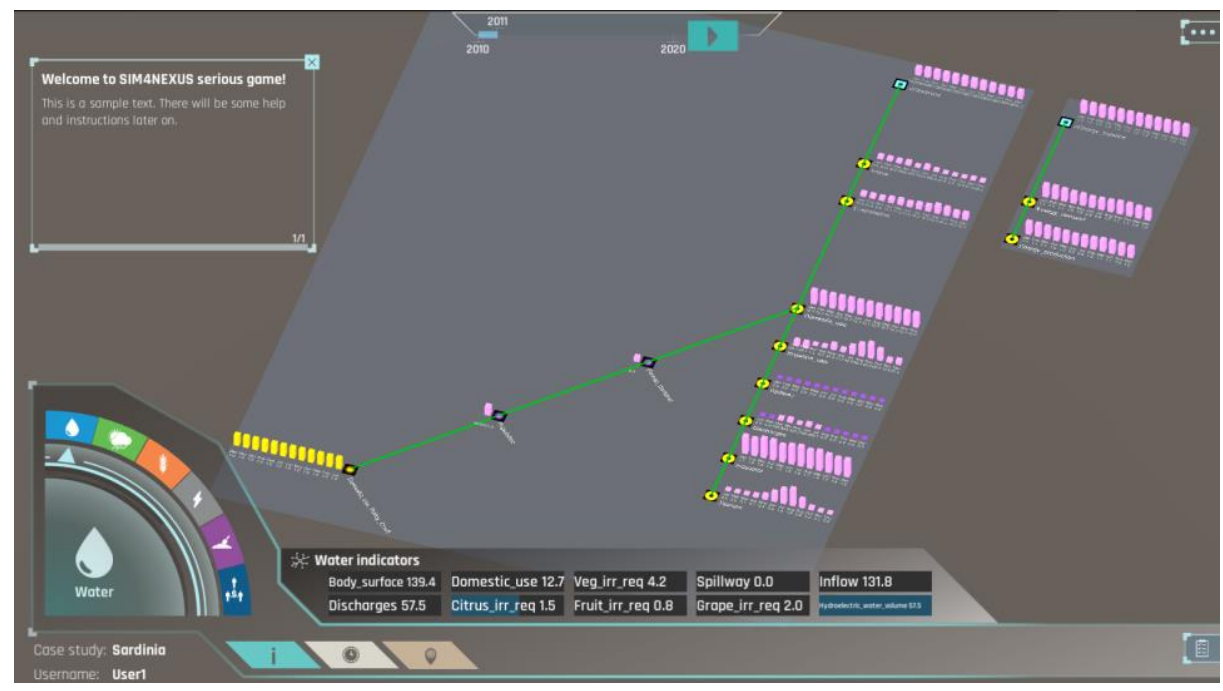

Figure 5: JSON Tree view of SDM policy run

\section{Discussion and Conclusions}

The work in this paper highlights how complex systems that depict the interactions between the land, food, energy, water and climate sectors within the nexus can be modelled against future climate scenarios. In contrast to silo based perspectives of policy impact analysis, the approach outlined here (that utilises thematic models within an SDM) allows for simulation and visualisation of the crosssector consequences/knock-on effects that given policies may have.

The incorporation of a developed SG to the frontend of the SDM allows for the complexities of the model to be hidden from policy makers and yet still provide guidance as to how policy changes will affect regions over time and what the underlying drivers to these policies are.

The methods and tools demonstrated within this work are being developed to be both transferable and scalable and models are currently being developed at regional, national, and transboundary levels with additional "higher level" case studies looking at continental and global perspectives.

Due to the variability in both locations and scales of the case studies being examined, it is envisioned that the work/methodologies being produced within this project will be transferable to other countries and/or regions and potentially prove to be a valuable tool to support cross collaboration between stakeholders whose work influences components of the nexus and help provide a more comprehensive understanding of the impacts polies have across different sectors and aid in efficient policy making for proactive responses to future climate change scenarios. 


\section{References}

CAPRI (Common Agricultural Policy Regionalised Impact Modelling System). (2017). Retrieved October 10, 2017, from http://www.capri-model.org/dokuwiki/doku.php

E3ME: Our Global Macro-econometric Model. (2017). Retrieved October 10, 2017, from https://www.camecon.com/how/e3me-model/

GTAP (Global Trade Analysis Project). (2017). Retrieved October 10, 2017, from https://www.gtap.agecon.purdue.edu/models/current.asp

Hempel, S., Frieler, K., Warszawski, L., Schewe, J., \& Piontek, F. (2013). A trend-preserving bias correction \&ndash; the ISI-MIP approach. Earth System Dynamics, 4(2), 219-236. https://doi.org/10.5194/esd-4-219-2013

Mereu, S., Sušnik, J., Trabucco, A., Daccache, A., Vamvakeridou-Lyroudia, L., Renoldi, S., ... Assimacopoulos, D. (2016). Operational resilience of reservoirs to climate change, agricultural demand, and tourism: A case study from Sardinia. Science of The Total Environment, 543, Part, 1028-1038. https://doi.org/http://dx.doi.org/10.1016/j.scitotenv.2015.04.066

Mohtar, R. H., \& Daher, B. (2010). Water, Energy, and Food: The Ultimate Nexus. In Encyclopedia of Agricultural, Food, and Biological Engineering, Second Edition (pp. 1-5). CRC Press. https://doi.org/doi:10.1081/E-EAFE2-120048376 